\title{
Consórcio de medicamentos no Paraná: análise de cobertura e custos*
}

\author{
Alide Marina Biehl Ferraes** \\ Luiz Cordoni Junior***
}

SuMÁrio: 1. Introdução; 2. Objetivos; 3. Metodologia; 4. Resultados; 5. Discussão e considerações finais.

SUMMARY : 1. Introduction; 2. Objectives; 3. Method; 4. Results; 5. Discussion and final remarks.

Palavras-chave: estratégia de gestão; cobertura de serviços públicos de saúde; custos de medicamentos; compras compartilhadas; economia farmacêutica; consórcio de medicamentos.

KEY WORDS: management strategy; public health service coverage; drug costs; shared purchases; pharmacoeconomics; drug consortium.

A redução de custos na compra de medicamentos é preocupação constante dos administradores públicos. Este artigo analisa a cobertura e custos do Consórcio Paraná Saúde (CPS), constituído para aquisição de medicamentos para prefeituras do Paraná. A cobertura abrangeu os municípios participantes e suas populações. Os custos dos medicamentos adquiridos foram comparados com os valores constantes no Banco de Preços do Ministério

\footnotetext{
* Artigo recebido em nov. 2005 e aceito em dez. 2006. Este artigo baseia-se na dissertação de mestrado em saúde coletiva de Alide Marina Biehl Ferraes, intitulada Política de medicamentos na atenção básica e a assistência farmacêutica no Paraná, defendida em 14 de agosto de 2002, UEL, Londrina, PR, Brasil. O trabalho conquistou o 1 lugar - categoria Mestrado - Prêmio de Incentivo em Ciência e Tecnologia para o SUS do Ministério da Saúde em 2002. Os autores agradecem carinhosamente à Deise Sueli de Pietro Caputo (diretora executiva do Consórcio Paraná Saúde) e à Lore Lamb (Cemepar) pela disponibilidade e acesso aos dados.

** Professora da Faculdade de Filosofia, Ciências e Letras de Cornélio Procópio. Endereço: Rua Primo Bozelli, 254 - Jardim Bandeirantes - CEP 86300-000, Cornélio Procópio, PR, Brasil. E-mail: ferraes@brturbo.com.br.

*** Professor do Programa de Pós-Graduação em Saúde Coletiva da Universidade Estadual de Londrina, Centro de Ciências da Saúde. Endereço: Av. Robert Kock, 60 - Vila Operária - CEP 86060-300, Londrina, PR, Brasil. E-mail: cordoni@sercomtel.com.br.
} 
da Saúde (BP/MS). Até o final de 2000, o CPS atingia 88,2\% dos municípios e $55,6 \%$ da população paranaense. Dos municípios participantes, 83,5\% possuíam menos de 20 mil habitantes. Foram comparados os preços de 55 itens constantes na lista de compras do CPS e no BP/MS em 2000. Destes, 46 apresentaram preços menores nas compras do CPS, um teve preço igual e oito apresentaram preços maiores. A aquisição pelo consórcio teve o custo de $\mathrm{R} \$ 332.397,70(29,7 \%)$ a menos do que custaria com os preços apontados no BP/MS. A constituição do CPS mostrou-se uma boa estratégia administrativa de farmacoeconomia, propiciando agilidade e racionalidade no uso dos recursos financeiros, possibilitando a ampliação do acesso da população aos medicamentos.

A drug consortium in Paraná: analysis of coverage and costs

Public administrators are always concerned in reducing the costs of drug purchases. This article analyzes the coverage and costs of the Paraná Health Consortium (CPS) which was created to purchase drugs for municipalities of the state of Paraná, Brazil. Coverage included the participating municipalities and their populations. The costs of the acquired drugs were compared to the values available in the Price Database of the Health Department (BP/MS). Until the end of 2000, the CPS had covered $88.2 \%$ of the municipalities and $55.6 \%$ of the Paraná population. Among the participating municipalities, $83.5 \%$ had fewer than 20,000 inhabitants. The prices of 55 items available on the purchase list of the CPS and in the BP/ MS were compared: 46 were lower, one was the same and eight were higher than those of the BP/MS. Acquisition by the consortium has cost R $\$$ $332,397.70$ (29.7\%) less than that by the BP/MS standards. The implementation of the CPS was found to be a good administrative strategy of pharmacoeconomics which led to agility and rationality in the use of financial resources, allowing wider access of the population to drugs.

\section{Introdução}

O Sistema Único de Saúde (SUS) é a forma constitucional brasileira de o cidadão ter garantido o acesso a ações e serviços de saúde, o que inclui a assistência farmacêutica (Brasil, 1990; Carvalho e Santos, 1995; Bermudez e Bonfim, 1999). O medicamento é um insumo importante para o bem-estar das populações e deve ser encarado como um meio, uma possibilidade a ser usada tanto para prevenir agravos, quanto também para recuperar a saúde. Nessa perspectiva, a ênfase deve ser no usuário do medicamento e devem ser pensadas ações individuais e coletivas para promover o acesso aos fármacos necessários (Ferraes e Cordoni Junior, 2002). Assim, as políticas de saúde devem ser pensadas com amplitude prevendo o direito da população de re- 
ceber os medicamentos necessários mediante compromissos no financiamento das esferas de governo (federal, estadual e municipal) e no gerenciamento adequado desses recursos.

Não é novidade que o setor da saúde enfrenta graves problemas de financiamento e que os preços dos medicamentos têm aumentado além da inflação no Brasil (Bermudez e Bonfim, 1999; Naves e Silver, 2005) e em outros países da América Latina (Homedes e Ugalde, 2005). Sabe-se também da grande dificuldade de acesso ao medicamento por parte do segmento que fica à margem do poder de consumo no país (Brasil, 1999b).

Se, por um lado, o acesso aos medicamentos é um direito da população e, por outro, os recursos são limitados, há necessidade de se compartilhar responsabilidades no financiamento entre as esferas de governo para não sobrecarregar ainda mais os municípios, que têm, atualmente, a maior parte das responsabilidades de prover ações e prestar serviços (Barros, 199-; Carvalho e Santos, 1995).

Visando normalizar a participação das três esferas de governo no financiamento da assistência farmacêutica (AF), o Ministério da Saúde (MS) emitiu a Portaria no 176/99 estabelecendo critérios e requisitos para habilitar municípios e estados ao Incentivo à Assistência Farmacêutica Básica (IAFB), incluído no Piso de Atenção Básica (PAB). Os valores para as três esferas de governo, totalizando $\mathrm{R} \$ 2,00$ por habitante/ano foram assim divididos: governo federal $\mathrm{R} \$ 1,00$, governo estadual, mínimo de $\mathrm{R} \$ 0,50$, e governo municipal mínimo de $R \$ 0,50$ (Brasil, 1999a). Segundo essa portaria, os recursos deveriam ser disponibilizados, a cada mês, na conta do Fundo Municipal de Saúde ou do Fundo Estadual de Saúde, de acordo com pacto realizado no âmbito da Comissão Intergestores Bipartite (CIB). As contrapartidas estaduais e municipais, definidas e aprovadas nessas comissões em cada estado, não poderiam ser inferiores ao repasse do nível federal (Brasil, 1999a, 2000b, 2001, 2002).

Existem quatro formas de pacto entre estados e municípios para aquisição de medicamentos com os recursos do IAFB: aquisição totalmente centralizada no estado, parcialmente centralizada no estado, totalmente descentralizada no município e parcialmente descentralizada no município (Brasil, 2000b, 2002). Essas formas de pacto foram amplamente discutidas por ocasião da I Oficina de Assistência Farmacêutica promovida pelo MS no Paraná (Brasil, 2000b). E, em 2002, publicadas pelo MS, estando assim definidas:

V totalmente centralizada no estado - nesta forma de pactuação, os recursos financeiros dos níveis federal, estadual e municipal são depositados no fundo estadual de saúde e o processo de aquisição de medicamentos é realizado pelo estado; 
> parcialmente centralizada no estado - os recursos financeiros dos níveis federal e estadual são depositados no fundo estadual de saúde, cabendo ao estado a aquisição e distribuição dos medicamentos pactuados aos municípios, nos prazos previamente definidos e nos valores correspondentes a esses recursos. Caberá aos municípios a aquisição de medicamentos em valores equivalentes às suas respectivas contrapartidas;

v totalmente descentralizada no município — os recursos financeiros dos níveis federal, estadual e municipal são depositados no fundo municipal de saúde e os medicamentos são adquiridos pelo município;

จ parcialmente descentralizada no município - os recursos financeiros dos níveis federal e municipal são depositados no fundo municipal de saúde e o estado faz aquisição dos medicamentos pactuados, em valores equivalentes à sua contrapartida, e os repassa aos municípios nos prazos previamente definidos (Brasil, 2002:9-10).

Pensando em alternativas para racionalizar e otimizar os recursos disponíveis para o IAFB, no Paraná, em 1999, de forma pioneira no Brasil, os municípios se articularam, e, em parceria com o estado, criaram um consórcio para aquisição de medicamentos básicos em escala denominado Consórcio Paraná Saúde (CPS) (Ferraes, Cordoni Junior e Castro, 2003).

No Paraná existem duas formas de pacto, podendo cada município optar por uma delas. O pacto que corresponde a parcialmente centralizada no estado, contempla os municípios que aderiram à estratégia do CPS, e a que corresponde a parcialmente descentralizada no município contempla os municípios nãoconsorciados (Paraná, 2000).

A avaliação de estratégias inovadoras como a adotada no Paraná é fundamental para sua validação, abandono e/ou correções.

\section{Objetivos}

Verificar a cobertura do Consórcio Paraná Saúde e comparar custos dos medicamentos comprados pelo consórcio em relação aos preços praticados pelo Banco de Preços do Ministério da Saúde (BP/MS).

\section{Metodologia}

Para verificar a cobertura do CPS no estado do Paraná foi utilizado o indicador: população atingida pelo programa em relação à população total. A população considerada para os cálculos foi a estimativa populacional 2000 da 
Contagem da População realizada pelo IBGE em 1996, utilizada pelo MS para o cálculo do PAB (Paraná, 2000).

Para verificar os preços praticados pelo CPS foi utilizada a Concorrência no 06/00, de 11 de dezembro de 2000, realizada pelo Consórcio Paraná Saúde, cujo resultado pode ser acessado em <www.consorcioparanasaude.com.br $>$. Os preços apurados foram comparados àqueles registrados no BP/MS em 2000 <http://dtr2001.saude.gov.br/banco/index.htm>.

Foram calculados os custos praticados pelo consórcio e pelo BP/MS de todos os itens de medicamentos constantes em ambas as listas.

\section{Resultados}

\section{Cobertura}

O Consórcio Paraná Saúde atingia, até o final de 2000 , a grande maioria $(88,2 \%)$ dos municípios do estado e 5.209 .467 habitantes, correspondendo à cobertura de $55,6 \%$ da população paranaense.

Os 47 municípios $(11,8 \%)$ que não aderiram ao CPS concentram quase a metade da população do estado e da contrapartida federal referente ao IAFB $(44,4 \%)$. Esses municípios recebem o recurso federal depositado direto no fundo municipal de saúde, sendo responsáveis pela sua administração na aquisição de medicamentos para atenção básica.

A tabela 1 demonstra a adesão e não-adesão dos municípios segundo faixas populacionais.

\begin{tabular}{|c|c|c|c|c|c|c|}
\hline \multicolumn{7}{|c|}{$\begin{array}{c}\text { Tabela } 1 \\
\text { Distribuição dos habitantes por grupos de municípios segundo } \\
\text { adesão ou não-adesão ao Consórcio Paraná Saúde } \\
\text { (Paraná, 2001) }\end{array}$} \\
\hline \multirow{2}{*}{ Habitantes $\mathrm{p}$ / município } & \multicolumn{2}{|c|}{ Adesão } & \multicolumn{2}{|c|}{ Não-adesão } & \multicolumn{2}{|c|}{ Total } \\
\hline & $\mathrm{N}$ & $\%$ & $\mathrm{~N}$ & $\%$ & $\mathrm{~N}$ & $\%$ \\
\hline$<10.000$ & 198 & 56,2 & 14 & 29,8 & 212 & 53,1 \\
\hline 10.001 a 20.000 & 96 & 27,3 & 12 & 25,5 & 108 & 27,1 \\
\hline$>20.001$ & 58 & 16,5 & 21 & 44,7 & 79 & 19,8 \\
\hline Total & 352 & 100,0 & 47 & 100,0 & 399 & 100,0 \\
\hline
\end{tabular}

Merece destaque que 26 municípios com menos de 20 mil habitantes não aderiram ao consórcio, sendo 14 municípios com menos de 10 mil habitantes e 12 com população entre 10.001 e 20 mil habitantes. 
Observando-se a tabela 2 , percebe-se que os 26 municípios com população inferior a 20 mil habitantes que não aderiram ao consórcio estão localizados em oito das 22 Regionais de Saúde (RS) do estado. Na 12 $2^{\text {a }}$ RS se encontram oito desses municípios. Os demais pertencem à $4^{\underline{a}}$ e $11^{\text {a }} \mathrm{RS}$, com quatro municípios em cada uma. Em relação aos municípios com população maior de 20 mil habitantes e que não aderiram ao Consórcio Paraná Saúde, a região metropolitana ( $2^{\mathrm{a}} \mathrm{RS}$ ) comporta o maior número deles (quatro). Nas demais RS geralmente o município sede da regional é que optou pela não-adesão ao Consórcio de Medicamentos.

\begin{tabular}{|c|c|c|c|c|c|}
\hline \multicolumn{6}{|c|}{$\begin{array}{l}\text { Tabela } 2 \\
\text { Quadro demonstrativo dos municípios do Paraná distribuídos por } \\
\text { Regional de Saúde, faixas populacionais e adesão ou não-adesão } \\
\text { ao Consórcio Paraná Saúde } \\
\text { (Paraná, 2001) }\end{array}$} \\
\hline \multirow{2}{*}{$\begin{array}{l}\text { Regionais } \\
\text { de Saúde } \\
(\mathrm{RS})\end{array}$} & \multirow{2}{*}{$\begin{array}{c}\text { Número de } \\
\text { municípios } \\
\text { por RS }\end{array}$} & \multicolumn{2}{|c|}{$\begin{array}{l}\text { Municípios com menos } \\
\text { de } 20 \text { mil habitantes }\end{array}$} & \multicolumn{2}{|c|}{$\begin{array}{l}\text { Municípios com mais } \\
\text { de } 20 \text { mil habitantes }\end{array}$} \\
\hline & & $\begin{array}{l}\text { Aderiram ao } \\
\text { consórcio }\end{array}$ & $\begin{array}{l}\text { Não aderiram } \\
\text { ao consórcio }\end{array}$ & $\begin{array}{l}\text { Aderiram ao } \\
\text { consórcio }\end{array}$ & $\begin{array}{l}\text { Não aderiram } \\
\text { ao consórcio }\end{array}$ \\
\hline $1 \underline{a}$ & 7 & 3 & - & 3 & 1 \\
\hline $2 \underline{a}$ & 29 & 16 & - & 9 & 4 \\
\hline 3 & 12 & 4 & 2 & 5 & 1 \\
\hline $4 \mathrm{a}$ & 9 & 3 & 4 & - & 2 \\
\hline 5 & 20 & 15 & - & 5 & - \\
\hline $6^{\mathrm{a}}$ & 9 & 7 & - & 2 & - \\
\hline $7 \underline{a}$ & 15 & 11 & - & 4 & - \\
\hline $8 \underline{a}$ & 27 & 22 & 3 & 2 & - \\
\hline ga & 9 & 6 & - & 2 & 1 \\
\hline $10^{\mathrm{a}}$ & 25 & 23 & - & 1 & 1 \\
\hline $11^{a}$ & 25 & 18 & 4 & 2 & 1 \\
\hline $12^{\mathrm{a}}$ & 21 & 12 & 8 & - & 1 \\
\hline $13^{a}$ & 11 & 8 & 2 & - & 1 \\
\hline $14^{\mathrm{a}}$ & 28 & 27 & - & 1 & - \\
\hline $15^{\mathrm{a}}$ & 30 & 22 & - & 7 & 1 \\
\hline $16^{a}$ & 16 & 14 & & - & 2 \\
\hline $17 \underline{a}$ & 20 & 14 & 2 & 2 & 2 \\
\hline $18^{a}$ & 22 & 19 & - & 2 & 1 \\
\hline $19 \underline{a}$ & 22 & 18 & - & 3 & 1 \\
\hline $20 \mathrm{a}$ & 18 & 12 & 1 & 4 & 1 \\
\hline $21 \underline{a}$ & 7 & 4 & - & 3 & - \\
\hline $22 \mathrm{a}$ & 17 & 16 & - & 1 & - \\
\hline Total & 399 & 294 & 26 & 58 & 21 \\
\hline
\end{tabular}




\section{Custos}

A Concorrência no 06, de dezembro de 2000, realizada pelo Consórcio Intergestores Paraná Saúde (2000) contou com 106 itens de medicamentos (correspondendo a $92,2 \%$ dos itens com que o consórcio trabalhava à época). No Registro do Banco de Preços/MS constavam 70 itens de medicamentos básicos (Brasil, 2000a). Foram analisados os preços de 55 itens de medicamentos constantes nas duas listas (correspondendo a 78,6\% dos itens da lista do Banco de Preços/MS e a 51,9\% da lista da Concorrência do Consórcio Paraná Saúde).

Pela comparação de preços praticados nas duas fontes analisadas, podese observar que os preços pagos pelo Consórcio Paraná Saúde para aquisição dos medicamentos são sistematicamente mais baixos do que os praticados pelo Banco de Preços do MS. A variação de preços é diferente para cada item de medicamento, e ocorre em 46 itens, variando de 0,7\% até 68,00\%.

A comparação entre os preços do Consórcio Paraná Medicamentos e do BP/MS realizada com cada um dos 55 itens disponíveis nas duas listas relevaram que a aquisição pelo Consórcio Paraná Saúde custou $\mathrm{R} \$ 785.856,59$ com estes itens. Se esta compra fosse efetuada com os preços referenciados no BP/ MS nos mesmos itens, o gasto seria de $\mathrm{R} \$ 1.118 .254,2$. A diferença de $\mathrm{R} \$$ $332.397,70$ corresponde a $29,7 \%$ a menos na compra do consórcio em relação ao que custaria com os preços praticados pelo BP/MS.

A tabela 3 apresenta a comparação do gasto em reais com os oito medicamentos adquiridos com preços superiores no Consórcio Paraná Saúde em relação ao BP/MS. A soma da diferença desses oito itens totaliza $\mathrm{R} \$ 13.286,667$. O Banco de Preços/MS nesses oito itens pratica preços 16,1\% menores do que o Consórcio Paraná Saúde para aquisição.

\begin{tabular}{|lccc|}
\hline \multicolumn{5}{|c|}{$\begin{array}{c}\text { Tabela } 3 \\
\text { Comparação demonstrativa do gasto em reais com oito }\end{array}$} \\
\begin{tabular}{l} 
medicamentos adquiridos com preços maiores no Consórcio \\
Paraná Saúde em relação ao Banco de Preços/MS \\
(Paraná, 2001) \\
\hline
\end{tabular} Consórcio Paraná Saúde & Banco de Preços/MS & Diferença \\
\hline Medicamento & $4.575,000$ & $2.699,250$ & $1.875,750$ \\
Clorpromazina 100mg comprimidos & $15.744,000$ & $9.446,400$ & $6.297,600$ \\
Fenobarbital 100mg comprimidos & 270,000 & 116,333 & 153,667 \\
Haloperidol 0,2\% gotas & $18.273,600$ & $16.920,000$ & $1.353,600$ \\
Neomicina + bacitracina pomada & & $1.035,000$ & $2.295,500$ \\
Prometazina 25mg & & & continua
\end{tabular}




\begin{tabular}{|lccr|}
\hline Medicamento & Consórcio Paraná Saúde & Banco de Preços/MS & Diferença \\
\hline Sais reidratação oral envelope & $27.984,000$ & $27.244,800$ & 739,200 \\
Sulfato ferroso 40mg comprimidos & $11.676,600$ & $11.227,500$ & 449,100 \\
Tiabendazol 500mg comprimidos & 432,000 & 309,240 & 122,760 \\
Total & $82.285,200$ & $68.998,523$ & $13.286,667$ \\
\hline Fontes: Brasil (2000a); Consórcio Intergestores Paraná Saúde (2000). & & \\
\hline
\end{tabular}

$\mathrm{O}$ valor da diferença desses oito itens adquiridos com valores superiores no consórcio (R\$ $13.286,667)$ corresponde a somente $4 \%$ do valor total da diferença entre os preços do consórcio e do BP/MS ( $\mathrm{R} \$ 332.397,70$ ).

\section{Discussão e considerações finais}

Sem dúvida o IAFB foi uma iniciativa importante para apoiar a assistência básica. Entretanto, seu valor é considerado muito baixo. A plenária da $12^{\mathrm{a}}$ Conferência Nacional de Saúde, realizada em 2003, referendando as recomendações da 1를 Conferência Nacional de Medicamentos e Assistência Farmacêutica, considerou como valor adequado $\mathrm{R} \$ 12,00$ por habitante/ano. O MS deveria custear $\mathrm{R} \$ 6,00$. Estados e municípios dividiriam igualmente o custeio dos outros R\$ 6,00 (Brasil, 2004:156).

Além disso, reconhece-se na administração pública brasileira o vício de se gastar mal. Na expressão de Carvalho, "tem-se pouco e gasta-se mal o pouco que se tem". 1

A iniciativa do Paraná caminha na contramão dessa tradição, buscando alternativa racionalizadora para a redução de custos, com potencial de se preservar a qualidade dos medicamentos adquiridos. Pelos resultados encontrados a experiência apresentou-se exitosa, embora com possibilidade de aperfeiçoamento. Trata-se da aplicação do conceito de "minimização de custos" da farmacoeconomia (Marin et al., 2003). Segundo Velázquez (1999a), a farmacoeconomia em países em desenvolvimento deve servir para a "racionalização de recursos existentes".

A grande cobertura populacional do consórcio, especialmente dos pequenos municípios, indica seu potencial como instrumento de promoção da

\footnotetext{
${ }^{1}$ Informação fornecida por Gilson Carvalho em palestra, "Entendendo o processo orçamentário", realizada em Curitiba para o Conselho Estadual de Saúde/Secretaria de Estado da Saúde do Paraná, em 7 de abril de 2003.
} 
eqüidade de acesso aos bens e serviços de saúde. Buss (1999:15) considera a questão dos medicamentos "um insumo crítico no processo de cuidados de saúde na América Latina" e, como tal, os governos devem buscar a eqüidade no acesso a tais insumos, criando mecanismos para acompanhar preços.

Joncheere (1997:55) menciona que:

muitos municípios estão comprando, mas seu poder de compra dilui-se e torna-se ineficiente para volumes inexpressivos e pela falta de ofertas. Têm sido sugeridos, por exemplo, sistemas alternativos de compra, com o propósito de combinar agilidade no processo de aquisição com economia de escala e controle comunitário tal como os financiamentos por consórcios de municípios.

Marin e colaboradores (2003) recomendam às administrações públicas a criação de mecanismos de ampliação da escala de compras pela adoção de compras consolidadas, sugerindo aquisições para períodos de 12 meses, por meio de associações ou consórcios entre várias instituições, processando compras em conjunto.

Entretanto, a literatura nacional e internacional não registra iniciativas de redução de custos mediante organizações associativas como os consórcios. Exceção feita a um curioso caso ocorrido na Argentina. O Instituto Nacional de Servicios Sociales para Personas Jubiladas y Pensionistas (INSSPJP), para congelar seus crescentes gastos com medicamentos para tratamentos ambulatoriais e de câncer, estabeleceu um repasse fixo mensal a um consórcio de indústrias farmacêuticas (destaques nossos) que se responsabilizaria pelo fornecimento dos medicamentos aos beneficiários do instituto. Tal iniciativa resultou num aumento médio dos preços dos fármacos de US\$16,73 em 1996 para US\$ 24,49 em 1999, ou 46\%. A diferença era paga pelos beneficiários do INSSPJP (Cervellino et al., 2003).

O Consórcio Paraná Saúde pode ser apontado como iniciativa bem-sucedida de uso racional de medicamentos. Para Velázquez (1999b) o gasto com o uso racional de medicamentos deve ser contabilizado como investimento social de grande utilidade na saúde e na redução de custos em longo prazo. O mesmo autor considera que a reforma do setor saúde deve enfocar garantia de acesso por meio de medidas e estratégias que promovam o uso racional de medicamentos, incluindo ética e economia.

O presente estudo detectou uma possível falha da estratégia do consórcio que foi a não-adesão de 26 municípios com população inferior a 20 mil habitantes, porém não logrou identificar as causas da não-adesão, que merecem ser averiguadas. 
Ressalta-se, ainda, que, independente do valor do recurso destinado à assistência farmacêutica e da esfera de governo que o gerencia, é imprescindível para a resolutividade dos serviços a garantia de compromisso dos gestores envolvidos, além da agilidade e racionalidade no uso de recursos. Aquisições bem-feitas no setor público, junto com as demais atividades do ciclo da assistência farmacêutica, contribuem para a credibilidade dos serviços farmacêuticos ofertados, e, numa visão ampliada, dos serviços de saúde de forma geral.

\section{Referências bibliográficas}

BARROS, E. O controle social e o processo de descentralização dos serviços de saúde. In: RODRIGUES NETO, E. et al. SUS coletânea de artigos. s.1.: s.n., 199-.

BERMUDEZ, J. A. Z.; BONFIM, J. R. de A. (Orgs.). Prefácio. In: a reforma do setor saúde. São Paulo: Hucitec, Sobravime, 1999. . Medicamentos e

BRASIL. Lei Federal n. 8.080, de 19 de setembro de 1990. Dispõe sobre as condições para promoção, proteção, recuperação da saúde, a organização e o funcionamento dos serviços correspondentes e dá outras providências. Brasília, DF, 1990.

Ministério da Saúde. Portaria n. 176, de 8 de março de 1999. Estabelece critérios e requisitos para a qualificação dos municípios e estados ao Incentivo à Assistência Farmacêutica Básica e define valores a serem transferidos. Brasília, DF, mar. 1999a.

. Ministério da Saúde. Secretaria de Políticas de Saúde. Departamento de Formulação de Políticas de Saúde. Política Nacional de Medicamentos. Brasília, DF, 1999b. 40p.

. Ministério da Saúde. Secretaria Executiva. Departamento de Programas Estratégicos em Saúde. Registro nacional de preços de medicamentos e correlatos: produtos disponíveis no Banco de Preços em Saúde. Brasília, DF, 2000a. Relatório extraído do sistema SAA da Subsecretaria de Assuntos Administrativos. Disponível em: <http:// dtr2001.saude.gov. br/banco/>.

. Ministério da Saúde. Secretaria de Políticas de Saúde. Assessoria de Assistência Farmacêutica. Como gerenciar bem o incentivo à assistência farmacêutica básica. Brasília, DF, 2000b. 27p.

. Ministério da Saúde. Secretaria de Políticas de Saúde. Departamento de Atenção Básica. Gerência Técnica de Assistência Farmacêutica. Incentivo à Assistência Farmacêutica Básica: o que é e como funciona. Brasília, DF, 2001. 25p.

. Ministério da Saúde. Secretaria de Políticas de Saúde. Departamento de Atenção Básica. Gerência Técnica de Assistência Farmacêutica. Assistência farmacêutica na atenção básica: instruções técnicas para sua organização. Brasília, DF, 2002. 113p. 
. Ministério da Saúde. Conselho Nacional de Saúde. In: CONFERÊNCIA NACIONAL DE SAÚDE - CONFERÊNCIA SÉRGIO AROUCA, 12. Relatório Final. Brasília, DF, 2004. 228p. Realizada de 7 a 11 de dezembro de 2003.

BUSS, P. M. Medicamentos na reforma do setor saúde: em busca da eqüidade na América Latina. In: BERMUDEZ, J. A. Z.; BONFIM, J. R. de A. (Orgs.). Medicamentos e reforma do setor saúde. São Paulo: Hucitec, Sobravime, 1999.

CARVALHO, G. I. de; SANTOS, L. Comentários à Lei Orgânica de Saúde (Lei 8.080/90 e Lei 8.142/90): Sistema Único de Saúde. São Paulo: Hucitec, 1995. 290p.

CERVELLINO, J. C. et al. Transferring the financial risks of pharmaceutical benefits from a large health care provider in Argentina to a consortium of pharmaceutical companies. Revista Panamericana de Salud Publica, Washington, v. 13, n. 4, p. 203-213, Apr. 2003.

CONSÓRCIO INTERGESTORES PARANÁ SAÚDE. Empresa vencedora: concorrência 06/ 12/2000. Curitiba, 2000. 3p.

FERRAES, A. M. B.; CORDONI JUNIOR, L. O medicamento, a farmácia, o farmacêutico e o usuário: novo século, novas demandas. Revista Espaço para Saúde, Londrina, v. 4, n. 1, dez. 2002. Disponível em: <www.ccs.uel.br/espacoparasaude/v4n1/index.htm>. Acesso em: 12 set. 2005.

; CASTRO, L. L. C. de. Paraná Saúde: consórcio de medicamentos básicos para os municípios. In: CONGRESSO BRASILEIRO DE SAÚDE COLETIVA, 7., 2003. Anais... Brasília: Abrasco, 2003.

HOMEDES, N.; UGALDE, A. Multisource drug policies in Latin America: survey of 10 countries. Bulletin of World Health Organization, Genebra, v. 83, n. 1, p. 64-70, Jan. 2005.

JONCHEERE, K. de. A necessidade e os elementos de uma política nacional de medicamentos. In: BONFIM, J. R. de A.; MERCUCCI, V. L. (Orgs.). A construção da política de medicamentos. São Paulo: Hucitec, Sobravime, 1997.

MARIN, N. J. et al. Assistência farmacêutica para gerentes municipais. Rio de Janeiro: Opas/ OMS, 2003. 334p.

NAVES, J. de O. S.; SILVER, L. D. Evaluation of pharmaceutical assistance in public primary care in Brasília, Brazil. Revista de Saúde Pública, São Paulo, v. 39, n. 2, p. 231-237, abr. 2005.

PARANÁ. Secretaria de Estado da Saúde. Plano estadual de assistência farmacêutica básica. Curitiba, 1999. 17p.

Secretaria de Estado de Saúde. Centro de Medicamentos do Paraná. Recursos federais e estaduais - assistência farmacêutica básica 2000. Curitiba, 2000. 22p. 
VELÁSQUEZ, G. Impacto económico del uso racional de medicamentos. In: BERMUDEZ, J. A. Z.; BONFIM, J. R. de A. (Orgs.). Medicamentos e reforma do setor saúde. São Paulo: Hucitec, Sobravime, 1999a.

Farmacoeconomia: evaluación cientifica o estratégia comercial? In: BERMUDEZ, J. A. Z.; BONFIM, J. R. de A. (Orgs.). Medicamentos e reforma do setor saúde. São Paulo: Hucitec, Sobravime, 1999b. 